\title{
Interactive Knowledge Construction in the collaborative building of an Encyclopedia
}

\author{
Philippe Lemoisson*, Stefano A. Cerri* \\ lemoisson@lirmm.fr ; cerri@lirmm.fr \\ *LIRMM, CNRS \& University Montpellier II \\ 161, Rue Ada - 34392 Montpellier Cedex 5, France
}

\begin{abstract}
$^{1}$
One of the major challenges of Applied Artificial Intelligence is to provide environments where high level human activities like learning, constructing theories or performing experiments, are enhanced by Artificial Intelligence technologies. This paper starts with the description of an ambitious project: EnCOrE ${ }^{2}$. The specific real world EnCOrE scenario, significantly representing a much wider class of potential applicative contexts, is dedicated to the building of an Encyclopedia of Organic Chemistry in the context of Virtual Communities of experts and students. Its description is followed by a brief survey of some major AI questions and propositions in relation with the problems raised by the EnCOrE project. The third part of the paper starts with some definitions of a set of "primitives" for rational actions, and then integrates them in a unified conceptual framework for the interactive construction of knowledge. To end with, we sketch out protocols aimed at guiding both the collaborative construction process and the collaborative learning process in the EnCOrE project.

The current major result is the emerging conceptual model supporting interaction between human agents and AI tools integrated in Grid services within a socio-constructivist approach, consisting of cycles of deductions, inductions and abductions upon facts (the shared reality) and concepts (their subjective interpretation) submitted to negotiations, and finally converging to a socially validated consensus.
\end{abstract}

Key words: Knowledge Construction, Learning Environments, e-Science, models for Human Learning

\section{Introduction}

This paper does not describe ongoing research concerned with the modeling, conception, design, realization or evaluation of a system tutoring something to someone under the verified hypothesis that teaching those concepts to those students is the proper thing to do. Rather, the research described here focuses on the preconditions for building such a system - or perhaps not building it at all - in a real world, semantically rich domain of extraordinary complexity (Organic Chemistry).

The rational analysis of those preconditions occurs under a strong assumption that underlies our work, i.e.: that learning in humans is a side effect of the autonomous construction of knowledge in a social context (social

\footnotetext{
1 "Work partially supported by the European Community under the Information Society Technologies (IST) programme of the 6th Framework Programme for RTD - project ELeGI, contract IST-002205. This document does not represent the opinion of the European Community, and the European Community is not responsible for any use that might be made of data appearing therein."

${ }^{2}$ EnCOrE : Encyclopédie de Chimie Organique Electronique (Computerised databases describing compounds exist, but have the same conceptual organization as the reference tomes from the 19th century, and are often misused; EnCOrE aims to address this problem)
} 
constructivism; situated learning; learning by doing in a social, dialectic environment; negotiation, convergence of jointly agreed ontologies).

In spite of the multiple support offered by the current literature on the plausible adequateness of social constructivism (Vygotsky 1978) very few concrete guidelines and reusable experiments have been published that offer a roadmap to follow, or architectural and behavioral patters to imitate. One exception, quite known by us, consists of DaNóbrega (2002), DaNóbrega et al. (2002) and DaNóbrega et al. (2003). These, however limited the explanation of the conceptual foundations to the toy domain of arches and the experimentation to a reduced subset of concepts in the domain of law. Our current research, instead, has the ambition to play a pivotal role within a full scale e-Science project: EnCOrE, where both descriptive and procedural concepts are in large number, wide complexity, crucial practical relevance - e.g.: for the synthesis of new drugs or the understanding of germane issues such as those in the domain of biotechnologies - and finally are described by a quite contradictory, partially ambiguous, totally dispersed and uncontrollable, rapidly growing technical and scientific documentation available online.

As Professor Alain Krief, founder of EnCOrE, expressed when addressing the ELeGI ${ }^{3}$ consortium:

"Currently the information is only delivered flat according to a single point of view dictated by tradition of "book organization" following the "Johannes Gutenberg age" (ca.1400-1468). In fact chemist brains work differently and the usual delivery message is context oriented. There is a huge number of different contexts which are covered and it is impossible using a book or even a lecture to describe them all (Experimental oriented, Starting material oriented, Product oriented, Mechanism oriented, Stereochemical oriented, Calculation oriented...).... Not only methods and tools needed for each context is different (Flasks, molecular models, Heavy calculation) but even the words used in each of these contexts are not properly defined.

The Construction of the EnCOrE Dictionary is extremely important for our project. It will fix the language and the related ideas and will play an important role in questioning EnCOrE. Its production is an act of power. If this power is not well understood, the chemists will ignore it. For that purpose it is extremely important that chemists accept and use it. For that purpose it should be elaborated through a collaborative work implying discussions, contextualization and consensus between the chemists community. We want to archive the discussions in order to keep the dictionary always alive by reactivating the discussions on a single word from time to time according to new needs.

We believe that the times where confirmed chemists, send by their respective governments were gathering in palaces sponsorized by IUPAC (Union of Pure and Applied Chemistry, http://www.chem.qmul.ac.uk/iupac/bibliog/gold.html) to build the compendium of chemical terms in a non contextual manner, is over."

\section{The EnCOrE project}

\subsection{Introducing Organic Chemistry}

Organic Chemistry ${ }^{4}$, as a part of Chemistry, produces its own objects of study through chemical reactions. Chemists are able to synthesize most of the compounds, even those which possess complex chemical structures including unknown ones, using rules, similarities with known facts and their own perception. Senior chemists are naturally willing to share their knowledge and to educate adequately the youngest both at the experimental and conceptual level. Because Organic Chemistry is intellectually highly structured, with many interconnected ideas, there are many quite different ways of teaching/learning it, and many possible starting points. But the end result is often the same - a broad understanding and a shared but to outsiders opaque language, with a serious problem in making the subject truly systematic. This is probably why senior chemists recognize difficulties in sharing their comprehension, especially in case of knowledge dissemination using electronic media. In fact building connections between the scientific experimental level and chemical equations, textual explanations and models in order to get an integrated expertise have not yet found an explicit expression.

These difficulties probably find their origin in several factors which lead to a set of ambiguous statements hard to exemplify and ineffective for knowledge sharing:

- A huge number of known substances in each single generic chemical reaction;

\footnotetext{
${ }^{3}$ ELeGI : European Learning Grid Infrastructure; official ELeGI Project URL http://www.elegi.org/

${ }^{4}$ Organic chemistry is the domain of sciences and technology which deals with the compounds containing Carbon, Hydrogen, some hetero-atoms (Oxygen, Nitrogen, Sulphur ...) and metals (Lithium, Sodium, Magnesium ...). Synthetic organic chemistry refers to the preparation of such compounds.
} 
- The necessity to talk about substructures restraining the vocabulary size to a reasonable magnitude while schemes are the only representation of adequate intricacy;

- A gap between the internal complexity of reactions (type and roles of reagents, solvents, catalysts, their ratio and concentrations, physical conditions, etc.) and the apparent simplicity of the chemical equation;

- The important influence of context on the meaning and on the understanding.

Meanwhile, the generalization of computer-assisted interactions in Society has a positive lever effect on the paradigm shift from knowledge transfer (product oriented computing) to interactive knowledge construction (service oriented computing).

As a consequence of these two facts, a project was launched: EnCOrE (Encyclopédie de Chimie Organique Electronique) as a result of an inter-disciplinary, international agreement involving several scientists and departments ${ }^{5}$. Further, the "human learning" aspects of EnCOrE have been considered at the core of a large integrated project approved and funded by the European Union under FP6: ELeGI (European Learning Grid Infrastructure) for the next 4 years. A related EU project on the $5^{\text {th }}$ FP: LEGE-WG ${ }^{6}$ offered us the scientific and technical background for the research we are describing, reported in the British Computer Society website ${ }^{7}$.

EnCOrE will provide complex information retrieval as an educational service in Organic Chemistry for university students and trainee researchers. EnCOrE will contribute to the design of new ways of teaching/learning, especially of Organic Chemistry, which has complex problems of having to handle words, chemical structures, mathematical formulae and experimental techniques, all equally important. It is also expected that, by revealing connections that are not currently recognized, it will even expose areas of ignorance that chemists are not aware of. As said by Guzdial et al. (1996), collaboration in complex problem solving activities facilitates both successful performance and reflection for learning (to enhance the learning process). A collaborative network between well-known synthetic chemists, computer chemists and computer scientists has been initiated in order to develop a productive collaborative environment.

\subsection{Main lines of EnCOrE internal architecture}

An Encyclopedia, when seen as a body of formally represented knowledge, relies on a conceptualization, in the meaning of an abstract, simplified view of the world wishing to represent something for some purpose (Gruber 1993). Notice that any conceptualization relies on a purpose; therefore it depends on a viewpoint. In the particular case of the EnCOrE project, harmonizing viewpoints at a very high semantic level is crucial. Relevant work has already been done inside the community of Chemical Scientists for establishing (i) a shared formal vocabulary (Lavoisier, 1789). (IUPAC 1997) and (ii) relations between the concepts (Reactions involve substances in the role of starting materials or products). According to Crubézy et al. (2003), Fensel et al. (2003), Gómez-Pérez (1999), Knublauch (2003), and Noy et al. (2000), this collective endeavour to achieve a shared understanding of information, formally expressed, and with a special focus on reusability, is commonly described as "ontology building".

In a way, ontologies already exist, are more or less formalized and may be available, but are not sufficient from a semantical point of view as they lack consistency and completeness, in other words they are a useful starting point but not the satisfactory result. In order to render this diagnosis more precise, let us consider the traditional difficulties encountered in ontology building:

As Klein and Fensel (2001) underline, the pieces of knowledge included in ontology are often not static, evolve over time: "ontologies are dynamic networks of meaning, in which consensus is achieved in a social process of exchanging information and meaning". The most difficult point considering ontologies is the management of their evolution, which should be considered as both an organizational and a technical process (Stojanovic et al. 2002). Modifications in ontologies (Noy et al. 2004) are caused by either: (i) changes in the domain, (ii) changes in the conceptualization, or (iii) changes in the explicit specification. In the case of EnCOrE, we may hope that the domain Organic Chemistry is rather stable. We also may hope that the language (Organic Chemistry) is strongly unified and is not subject to translations, which means that we have not to bother too much about changes in the explicit specification. Therefore the remaining cause of instabilities is (ii): changes in the conceptualization; for instance the chemical notion of "functional group" may be understood to characterize NOT the molecules themselves, but the relations between molecules: this has practical consequences in the

\footnotetext{
${ }^{5} \mathrm{http}: / /$ www-encore.enscm.fr/

${ }^{6}$ Official LEGE-WG Project URL (http://www.lege-wg.org/)

${ }^{7} \mathrm{http}: /$ ewic.bcs.org/cgi-bin/search.pl?p=1\&lang=en\&include=\&exclude=\&penalty $=0 \&$ sort $=\&$ mode $=$ all \&q=lege-wg
} 
ontology's structure. And difficulties arise when modification in one part of the ontology generate inconsistencies in other parts of the same ontology; in ontology-based instances as well as in depending ontologies and applications (see Fensel in [Stojanovic et al. 2002]).

We are fully aware of the fact that we have to control these changes in the conceptualization in a very accurate way since they have not been administered in an "ontology evolutionary process" for years; one of the hard tasks of EnCOrE is to unify "a posteriori" a great number of independent local evolutions in conceptualization, the expression of which may only be found in the scientific literature. We shall see that, in order to deal with this major difficulty, the conceptors of EnCOrE intend to:

- explicitly take into account viewpoints as it will be explained further in this chapter;

- rely on the strong assumption that each viewpoint is built during a conversational process by generalization upon duly described chemical experiments, and therefore will always remain relevant (under an implicit hypothesis of stability concerning the basic vocabulary of Organic Chemistry, while we deal with the elaboration of high level concepts) what is to be expected is the addition of new viewpoints, not the deletion of old ones. This seems to simplify the scenario.

We consider three principal components to construct an encyclopedia of Organic Chemistry:

a) An ONTOLOGICAL NUCLEUS containing domain-specific scientific terms defined and organized with explicit relations between them. On a consensual basis, terms will describe shared concepts ${ }^{8}$ using typical "Semantic Viewpoints" aimed at classifying theoretical situations, and therefore at contextualizing the meaning. In the same time, a collection of "Pragmatic Viewpoints"10 will be stored and managed in order to take into account the questions raised during the knowledge acquisition process;

The roles of the ontological nucleus are:

- to gather the shared vocabulary of the community, to explicit formal representation modes;

- to achieve a coherent integration of contents for their reusability and their recovery by Artificial Agents;

- to serve as a reference for those willing to construct new ontologies around it, by providing a reusable shared conceptual framework to describe meaning.

b) An EXPERIMENTAL CORPUS containing chemical reactions stored with their original description represented using terms and viewpoints from the ontological nucleus. The experimental corpus is a collection of facts in their experimental description based on the notion of Vessel Reaction ${ }^{11}$. The value of the experimental corpus will reside in the selection of reaction examples following a chart defined by EnCOrE editors, and in its data structure refereeing to the ontological nucleus. Experimental facts represent the "hard" reality less prone to controversy, and are a good basis to construct a stable ontological nucleus that will be reused in the theoretical corpus.

c) A THEORETICAL CORPUS made of scientific articles conceptualizing the experimental corpus. Article content will be "indexed" by the terms and viewpoints of the ontological nucleus. Based on the hard reality of the experimental corpus, the theoretical corpus will be the ground to construct a soft reality, in line with the Popperian approach of conjecture/refutation (Popper 1959). The semantic viewpoints and pragmatic viewpoints registered inside the ontological nucleus will help formalizing the Synthetic Organic Chemistry theories.

\footnotetext{
${ }^{8}$ Chemistry concepts : pure substance, chemical element, structure, functional group, vessel reaction, chemical equation, named reaction, retro-synthesis, etc ...

${ }^{9}$ Semantic Viewpoints : Equation/Redistribution, Taxonomy/Examples, and Strategy/Practice

${ }^{10}$ Pragmatic Viewpoints : Strategic, Stereo chemical, Reactivity

${ }^{11}$ Vessel Reaction: In a vessel under given physical conditions, when contacting chemical substances induces a chemical transformation, if a product can be isolated or if it is possible to show chemically the presence of an intermediate (by inducing an alternative reaction, orthogonally to the synthetic scheme), then a vessel reaction has been performed. Within one experiment, zero, one or several successive vessel reactions lead to isolation of created or recovered compounds
} 


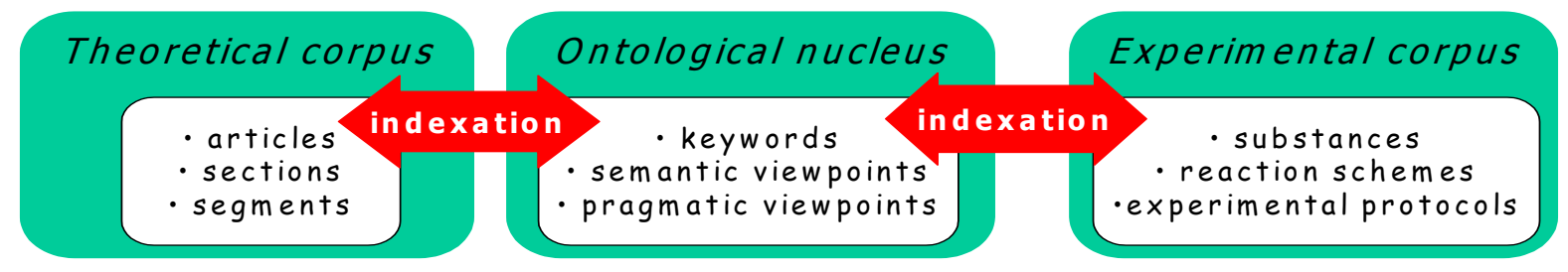

Figure 1 : Indexation relationship between the ontological nucleus and both the theoretical and experimental corpuses

Figure 1 shows the components of the encyclopedia, and the relations between them. The ontological nucleus plays the role of hard kernel, structuring the whole encyclopedia and very closely connected to scientific experimentation depicted in the experimental corpus; it contains the consensual and stable community representations. The theoretical corpus plays the role of peripheral soft layer pertaining to specific areas (articles, sections, segments) containing the controversial and variable representations based on the core ones: controversies may be due to group's assumptions (schools of Chemistry sharing a yet unexpressed viewpoint) or individual's assumptions. Its building block is the encyclopedia "article"; while the "segment" is defined as an editorial "atom", to which pragmatic and semantic viewpoints are attached. Each article is composed of "sections", themselves composed of segments indexed by both pragmatic and semantic viewpoints; therefore, the organization of the domain into formal structures will occur on a bi-dimensional way, corresponding respectively to the encyclopedia and dictionary modality of utilization of EnCOrE. Articles, as well as experimental protocols, include some terms of the ontological nucleus and the indexation link is established with the definition of the term in accordance with the relevant semantic viewpoint.

\subsection{Building the encyclopedia}

The preliminary construction of a consensual ONTOLOGICAL NUCLEUS will be a critical task. We foresee a collaborative construction process among a few established Organic Chemists, specialists in the area of synthesis, in order to secure from the beginning both the birth and the growth of the nucleus. The ontological nucleus will play the role of a valuable model for more effective work involving a larger number of actors, each of them submitting theoretical hypotheses to the validation of the group. Iterative control of the consequences of conceptual choices at a rational level will be essential. The challenge of this initial work will consist of the choices on its granularity and the convergence of the consensus among experts. Its construction will involve collaborative interactions over a shared dual reality based on the collection of experimental facts and pieces of scientific literature, as it will be exposed in Chapter 5. Shared concepts will be identified and named in reference to the shared reality, maintaining the semantic track and the consensus constraint. The identification and use of semantic viewpoints in the dictionary will help resolving conflicts over meaning of terms, by allowing the contextualization of term definition.

Pragmatic viewpoints will be identified and named while classifying generic questions. These questions will allow authors to organize their writing and to label their texts and schemes with explicit tags that will be recognized by searching (artificial) Agents. We claim that the explicit classification of pragmatic viewpoints by authors will play a key role in their learning process: not only learning Chemistry, but also learning how to perform meta-reasoning on Chemistry by using the suite of tools available in EnCOrE. The relation between generic questions and pragmatic viewpoints is exemplified in the following table 1:

Table 1 - Generic Questions \& Pragmatic Viewpoints

\begin{tabular}{|l|l|}
\hline Generic Question & Pragmatic Viewpoint \\
\hline What makes the chemical reaction $X X X$ so useful in synthesis? & Strategic \\
\hline What is the stereo chemical outcome of the reaction $X X X ?$ & Stereochemistry \\
\hline What is the reactivity of $X X X ?$ & Reactivity \\
\hline How can one obtain $X X X ?$ & Synthetic \\
\hline What are the conditions to transform $X X X$ into $Y Y Y ?$ & Conditions \\
\hline
\end{tabular}


Building the EXPERIMENTAL CORPUS is deeply connected to the capture of functional knowledge into an observational language. Even if "hard facts" are to be considered somehow "true", a technically profound dialectic process on their interpretation by experts is considered to be the major source of advancements in the chemical science itself offering an opportunity to established scientists participating to EnCOrE to profit from the process of organizational learning that will be necessary in order to reach a consensus and consolidate its results on the evolving Encyclopedia. In order to establish a solid basis for such an interpretation, an exploration of the common language used by chemists to describe what they do is necessary; and also a deep study of how elementary tasks are integrated into "experimental workflows".

This part of our work will have to take into account recent work combining ontological and knowledge engineering initiatives, like the ontological framework developed in (Yoshinobu 2004), or the approach described in (Chen et al. 2003) based on a framework and its implementation towards the delivery of knowledgebased service composition, or more generally, resource synthesis, in a web-enabled or Grid computing environment. This kind of approach, 'offering a democratized access to computing resources' will make EnCOrE a good application of the Grid, e.g. for providing access to molecular modeling tools (conformations, energy levels and geometry of frontier orbitals, etc.) without the need to install them.

\subsection{A “design for learnability” approach}

Any scientific construction makes sense only as much as it may be communicated, shared, learned, used by others; as much as the concepts and processes described and exemplified are reusable by others thanks to their understandability and learnability by other humans, both experts and beginners. In this sense, EnCOrE is aimed to become an "intelligent tutoring system" or, more precisely, an "intelligent learning environment", where chemists may engage in constructive interactions, thus "learn by doing, in the socially dialectic context of pairs". As expressed in Crozat et al. (2000): "our ontology, even if only a restrictive and partial representation of the domain, can bring standardization and methodology, providing to the actors of the domain a common language and agree on what is or should be a tutoring system".

Therefore from the beginning we foresee learnability by peers to be crucial in EnCOrE. We adopt in EnCOrE a clean "design for learnability" approach. It consists firstly of the support for the dynamic aspects of its interactive construction which will imply both senior and junior chemists. The approach of learning is here a synergic approach, sometimes called "human centered design", very different from the classical, applicative approach. Considering the junior chemist at the center, learning is clearly a social, constructive phenomenon, where knowledge acquisition happens through a process of subjective construction starting from the experience rather than through the discovery of an ontological reality (Bravo et al. 2002; VonGlasersfeld 1978). It occurs as a side effect of interactions, conversations and enhanced presence in the dynamic "Virtual Community" of the Encyclopedia authors and users.

Because of this approach, we shall have to define protocols for the collaborative aspects: from a modeling and computational viewpoint, our major challenge is not in controlling versions of the consolidated knowledge within the encyclopedia (the static aspects of the EnCOrE are out of the scope of this paper), but rather controlling and facilitating the conversations among agents (human as well as artificial) sharing the ambitious goal of building together a high level formal representation supporting theories in Organic Chemistry. Therefore, our challenge is twofold:

1. to enhance the communication and therefore the collaboration between Chemists

2. to allow the whole EnCOrE system to behave like a unified Service provider aimed at Scientific information management, storage and retrieval

This challenge, especially its second term which is the major topic of this paper, fits with the "semantic Grid" proclamation: the transition from a product view to the service view of the network supporting global interactions among human and artificial agents (Lemoisson et al. 2005; Cerri et al. 2005; Cerri 2005; Colaux-C et al. 2005).

This challenge tackles some well-known questions, among which:

- What are the critical processes underlying human scientific activity? 
- To what extent can "Artificial Agents" encapsulated in Grid Services ${ }^{12}$ take part in those processes?

- How can "Artificial Agents" encapsulated in Grid Services enhance those processes in order to improve human collaboration?

The following chapter is dedicated to a survey of those questions. Then we shall draw a conceptual framework for human and artificial agents in interaction. And finally we shall sketch out protocols aimed at enabling the interactive knowledge construction processes.

\section{A brief survey of some underlying theoretical questions}

\subsection{The process of "formal representation building" in human scientific activity}

From the EnCOrE objectives, we can understand that although Chemistry benefits from a structured language for describing compounds, this language is not sufficient for sharing high level knowledge about Chemical reactions. Representing dynamic transformations in order to build theories upon them is indeed a demanding task! This generic question of providing a simplified vision of reality through "formal representations" supporting "theories", respectively at the experimental and at the theoretical levels, has always been crucial in Science:

- from Duquenne (1999) referring to Piaget (1968), we understand that a major difficulty in experimental interpretation is the building of the appropriate formal language itself “ . .. it is therefore much more difficult to ascertain facts and to analyze them than to think or to deduct, and here is why experimental sciences were born long after deductive disciplines, the latter playing the role of framework and necessary conditions for the former, but in no way being sufficient ..."

- the French philosopher and epistemologist G. Bachelard (1975) clearly expresses the same need of a proper representation for any scientific activity: "drawing phenomenon and ordering into series the key events of an experiment, here is the first task where a scientific mind can assert itself. It is indeed by this way that one reaches the represented quantity, in between the concrete and the abstract, in an intermediary area where the mind pretends to conciliate mathematics and the experiments, laws and facts."

- this building of formal representations from observations implies complex non-monotonic psychological and sociological processes; this appears when we apply Piaget's theory to the underlying "cognitive structures"13: "Cognitive structures change through the processes of adaptation: assimilation and accommodation. Assimilation involves the interpretation of events in terms of existing cognitive structure whereas accommodation refers to changing the cognitive structure to make sense of the environment" (available at://tip.psychology.org/piaget.html). Those processes are the core of Karl R. Popper's model of theory building; they are driven by refutation and consensus, not by goal-achievement as in classical cooperation: "Science does not rest upon solid bedrock. The bold structure of its theories rises, as it were, above a swamp. It is like a building erected on piles. The piles are driven down from above into the swamp, but not down to any natural or "given" base; and if we stop driving the piles deeper, it is not because we have reached firm ground. We simply stop when we are satisfied that the piles are firm enough to carry the structure, at least for the time being (Popper 1959)".

Therefore, the question of describing facts and discovering theories has to be examined in the light of complex dynamic processes involving the whole community of experts of a given domain. The question we raise now is: "Can artificial agents, aimed at being encapsulated in Grid Services, play a useful role in those processes, and how?"

\subsection{The "model inference problem": to which extent can "Artificial Agents" take part in the discovery of formal representations?}

\subsubsection{Turing Machines inferring theories from facts}

\footnotetext{
${ }^{12}$ we use the expression Grid "Service" mainly in the meaning of "passive, user controlled facilities obeying to Grid standards"; whereas true "Services" would require dynamic components able to take initiatives

${ }^{13}$ patterns of physical or mental action that underlie specific acts of intelligence
} 
A remarkable attempt to use Turing Machines in order to infer theories from facts can be found in one of the most famous inheritors of Turing tradition (Shapiro 1991), who tackles the "model inference" problems:

"A model inference problem is an abstraction of the problem faced by a scientist, working in some domain under some fixed conceptual framework, performing experiments and trying to find a theory capable of explaining the results. In this abstraction the domain of inquiry is the domain of some unknown model $\mathrm{M}$ for a given first order language L, experiments are tests of the truth of sentences of $\mathrm{L}$ in $\mathrm{M}$, and the goal is to find a set of true hypotheses that imply all the true testable sentences. The main result of this paper is a general, incremental algorithm for solving model inference problems, which is based on the Popperian methodology of conjectures and refutations. The algorithm is shown to identify in the limit ${ }^{14}$ any model in a family of complexity classes of models... As part of the general algorithm, an algorithm for backtracing contradictions was discovered. This algorithm is applicable whenever a contradiction occurs between some conjectured theory and the facts ... it can trace back a source for this contradiction, namely a false hypothesis, and can demonstrate its falsity by constructing a counterexample to it"

Going through the whole algorithm is clearly out the range of this paper. It can be sketched in the following display:

Let $\mathrm{T}$ be the conjecture (a set of hypotheses):

Set $T$ to $\{$ empty sentence\}

Repeat

Read the next fact

Repeat

While the conjecture $\mathrm{T}$ is too strong do apply the contradiction backtracing algorithm, and remove from $\mathrm{T}$ the refuted hypothesis

While the conjecture is too weak do add to T refinements of previously refuted hypotheses

Until the conjecture $\mathrm{T}$ is neither too strong nor too weak

Forever

Although it may seem somewhat technical, we summarize in six points the successive hypotheses made by the author:

a. a first order language in clausal form L, with finitely many predicates and function symbols. Basically, Shapiro's approach consists in browsing in a sophistically optimized way through an enumerable set of possible theories, until one is found which is a proper model of a given set of observations; yet part of the difficulty is avoided in order to guarantee a result in a finite time

b. a subset $\mathrm{L}_{0}$ of $\mathrm{L}$ : the observational language

c. a subset $\mathrm{L}_{\mathrm{h}}$ of $\mathrm{L}$ including $\mathrm{L}_{0}$ : the hypothesis language .As the author outlines : "it should be noted that the assumption that the hypothesis language is fixed avoids one of the major problems in scientific discovery, which is the invention of a new conceptual scheme. Using the terminology of (Khun 1970), the model inference problem resembles more the puzzle-solving activity of "normal science", than the search for new conceptual schemes that is characteristic of "paradigm shifts" periods.

d. an oracle for the unknown model $\mathrm{M}$, that when given an observational sentence returns true if it is true in $\mathrm{M}$, false otherwise ${ }^{15}$.

e. an "admissibility requirement" has to be made on $\mathrm{L}_{0}$ and $\mathrm{L}_{\mathrm{h}}$, which "essentially says that $\underline{\mathrm{L}}_{0} \underline{\text { must }}$ contain enough information to refute any false theory that attempts to be $\mathrm{L}_{0}$-complete. Ehud Shapiro comments this point by saying: "the admissibility requirement is a necessary, but not sufficient condition for the solvability of a model inference problem ... to understand the concept of a palindrome (a string being the reversal of itself), a person must know first the concept of string reversal. Terms that denote such concepts are called theoretical terms and have to be known to, or built into the inference algorithm as "theoretical concepts".

f. a condition about $\mathrm{M}$, so that the check of a fact against a conjecture is bound in complexity (it is in general undecidable). This expresses the fact that the algorithm will infer "not too complex" models, which also means that in will infer models in "reasonable" time.

\footnotetext{
${ }^{14}$ Referring to (Gold, 1967), Ehud Shapiro explains: “... the most one can expect of an inductive inference algorithm is that after examining a finite number of facts about the model and making a finite number of wrong conjectures, such an algorithm will correctly conjecture a finite set of hypotheses true in the model, which imply all true observational sentences."

${ }^{15}$ it appears here that what people in the Turing tradition call "oracles" is a model of what experimental sciences call "experiments".
} 
From those strong hypotheses, we can understand that although Shapiro's tool is very powerful, it remains a tool "looking for solutions within a human pre-defined approach (see the author's comment in e. about theoretical concepts)".

\subsubsection{Is it possible to mechanize scientific discovery?}

Here is the ultimate question hiding behind our search for help in the discovery of formal representations...

Mario Alai from which a long quote is given in annex (Alai 2004), describes several attempts in this direction and gives us the following summary of his exploration, in which it appears that "scientific discovery", seems too ambitious a goal for artificial agents alone (at least until now):"the programs written by Simon's group, indeed, infer famous laws of physics and chemistry; but having found no new law, they cannot properly be considered discovery machines. The programs written in the "Turing tradition", instead, produced new and useful empirical generalization, but no theoretical discovery, thus failing to prove the logical character of the most significant kind of discoveries ... Admittedly, the "Holland et al" program PI has not (yet) made any new discovery, nor any old one as complex and important as those achieved by Simon's programs."

Pat Langley, surveys in Langley (2000) research about automated scientific discovery in the past two decades through five distinct types of discovery activity:

- $\quad$ building taxonomies

- discovering qualitative laws

- discovering quantitative laws that state mathematical relations among numeric variables

- $\quad$ building structural models that incorporate unobserved entities

- building process models which explain phenomena in terms of hypothesized mechanisms that involve change over time

He then identifies the major steps during which developers or users can influence the behavior of a computational discovery system:
problem formulation
representation engineering
date manipulation
algorithm manipulation
filtering and interpretation after algorithm invocation

His conclusion, after a detail examination of published results covering each type of discovery activity in several domains, is that human computer cooperation is a major factor and the key of success.

According to our survey, it seems that the most powerful attempts to enhance the building of "formal representations" with the help of artificial agents are a clever combination of human intelligence and sophisticated algorithms. Those algorithms rely on two "primitives" in which human intelligence is easily beaten by machine power:

- $\quad$ exhaustive exploration of a huge domain of possibilities

- testing of each possibility through deductive inference according to a set of rules

Emphasizing on the cooperation between human and machines uploads the third aspect of our question: "through which kind of protocols can artificial agents enhance human collaboration"?

\subsection{How can "Artificial Agents" enhance human collaboration?}

In this paragraph, we are going to present two examples of environments which seem very encouraging for our current challenge of supporting global interactions among human AND artificial agents for knowledge building and learning purposes.

\subsubsection{Discovery Learning Environments}

As a first example of such environment, we can find in DaNóbrega et al. (2003) the design and usage of Discovery Learning Environments “... within which learning events occur as a consequence of contradiction detection and overcoming, during human/machine cooperative work. We firstly introduce a model of an agent capable of handling such an approach of learning, by highlighting the exchanges that the agent should promote with a human learner. The model lies on the basis of the scientific rationale, particularly the empirical approach guided by the theory-experiment confrontation." 
The approach consists of the following steps:

- hierarchically organizing a vocabulary in order to describe the "objects" to learn from, for instance through the use of hierarchical labeled graphs. This is done by the Human Agent.

- constraining this vocabulary, which consists in :

- describing examples to the artificial agent

- activating Machine Learning so that the artificial agent proposes a set of constraints

- filtering the learnt knowledge (the artificial agent Axiomatics) according to what the human agent thinks pertinent

- testing and revising by proposing new "objects" to the artificial agent. Inadequacy of an object declared by the artificial agent lies on the basis of a contradiction revealed while the agent confronts the object description with the current Axiomatics. A revision process should then take place in order to reach a coherent behavior for the artificial agent. Such a revision process would require, however, the Human Agent to know how to reestablish the coherent status of the theory. As this is not always evident, before such a revision process could take place, the Human Agent may need to find out why a contradiction arises and therefore asks the artificial agent the reasons of its judgment.

\subsubsection{Building a Student Model}

A second example is available in Cerri et al. (1990), where a methodology and a software system for the gradual elaboration of a generative student model in some domain is described. Cerri emphasizes on a cyclic refinement process involving an expert, the tutorial/diagnoser and students, all linked through the environment. The aim is to overcome the problems related to the formulation of an enumerative theory of bugs, which is always very labor-intensive. The basic steps of this methodology are the following:

a. definition of the formal knowledge-base representing the field of study

b. construction by a domain specialist of an initial set of plausible bugs in terms of a perturbation of the correct domain model.

c. refinement and extension of the set of bugs by means of a series of Inverted Dialogues, using the models developed in stages $a$. and $b$.

d. use of the full set of validated bugs in the final tutoring system

"Step c.", based on Inverted Dialogues, is aimed at "allowing the expert to inspect and verify some of the initial set of error models proposed by the system designer. To achieve this, the expert must first identify the misconception represented by the model (from the errors that it generates), and then confirm that he/she has correctly identified and understood the misconception. Models verified in this way can be accepted for inclusion in the final version of the tutoring system.

- $\quad$ the first stage, identification of the misconception, is realized by an inverted dialogue in which the expert assumes the teacher's role, and examines the responses produced by the model. This should lead the expert to formulate some hypotheses about the misconceptions underlying the surface errors ...

- $\quad . .$. so that in the second stage of the dialogue, in which he experts plays the part of a student answering questions put by the system, he/she may simulate those errors. When the expert disagrees with the system, it indicates that the two participants in the dialogue are using different models. What we would like to do is to allow the system to acquire the model that is being used by the expert."

In each of the two above approaches, a purely logic-driven mediator is embodied in a Turing Machine, and appears a very powerful enabler of human collaboration in the Popperian cycle of predictions and refutations. Roughly speaking, a hard kernel (the logic mediator) is of great help for testing and refining a soft evolving theoretical consensus.

\subsection{Key lessons summary}

From this brief survey, we should like to outline a few points that will influence the next chapters:

- $\quad$ However sophisticated, a pure artificial intelligence cannot assume the critical tasks involved in the building of formal representations: no Grid Service can be expected to embody this crucial function. Although logic is the foundation of knowledge representation and plays an essential role in scientific activity, it is very doubtful that there exists a rational model for a "logic of discovery" which would 
lead to mechanization of scientific activity; therefore the human capacity to produce new testable hypotheses remains the key aspect in knowledge construction. Turing Machines are efficient, if not irreplaceable, for implementing duly specified theories or models within the framework of an embodied logic, linking hypotheses in a "non-defeasible way". They can even, in the case of Shapiro's approach, browse through an enumerable set of possible theories, until they find one which is a proper model of a given set of observations; but again, the target shape of those theories has to be given "a priori", and some conditions have to fulfilled in order to guarantee a result in a finite time. As Mario Alai emphasizes in (Alai 2004), "a serial computer cannot discover structures or mechanisms genuinely unpredictable at the moment of its programming".

- Even a connectionist network of artificial agents cannot pretend today to be as successful as human mind for the production of new testable hypotheses. Approaches like the one described in Holland et al. (1986), implementing competing parallel computations ruled by a selection based on "market laws", are raising more and more of interest today, yet it seems that they are far from being as powerful as human mind.

- Hopefully, leaving the "machine-centric" viewpoint and setting a unified framework where human as well as artificial agents interact for collaborative construction seems very promising! Examples can be found for elaboration of a constrained domain specific vocabulary (DaNóbrega et al. 2003) or elaboration of a student model (Cerri et al. 1990). A purely logic-driven mediator embodied in a Turing Machine, and playing the role of an artificial agent which will never express new ideas in the conversation, but will never make mistake in inferring from input given to it, appears a very powerful way of enabling human collaboration in the Popperian cycle of predictions and refutations. Such artificial logic-driven mediators, embodied in Grid Services, may take a decisive part in the collaborative building of formal representations. In order to specify the protocols associated to those mediators between Scientifics and artificial agents, we first need a unified "framework".

\section{A framework for the interaction of "Rational Agents"}

When specifying the EnCOrE Encyclopedia in chapter1, we have expressed the challenge of "providing a unified educational service in Organic Chemistry", by means of collaborative construction of an ONTOLOGICAL NUCLEUS linking together a THEORETICAL CORPUS and an EXPERIMENTAL CORPUS. This collaborative construction is to happen through conversational processes between authors, users and Grid Services.

We wish to build a "framework" where formally defined protocols can be run between human agents and artificial agents. This framework, already been presented in (Lemoisson et al. 2005), is much inspired by the multi-agents paradigm (Ferber 1999), and addresses collaborative scientific activity through the following steps:

- giving informal definitions of logical "primitives" for rationality

- connecting together the notions of "rationality" and "interaction"

- testing the "model" against ontology collaborative building

- testing the "model" against theory building

- sketching out a general framework

\subsection{Informal definitions of logical "primitives" for rational activity}

Coming back to the underlying processes supporting scientific activity, we need to provide some informal definitions for a few "technical" words that we are going to use, namely induction, abduction and deduction.

\subsubsection{Induction}

To start with induction, let us proceed chronologically and first refer to John Stuart Mill (1843), who insists on its creative aspect: "Induction is an inferring process; it starts from the known towards the unknown, and any operation which does not imply an inference, any process in which what seems to be the conclusion does not go beyond the initial premises, should not be called induction".

In Peirce (1955), we find a more fine-grained definition, where the notions of statements and laws appear: " a common feature of all kinds of induction is the ability to compare individual statements : using induction it is possible to synthesize individual statements in general laws - inductive generalizations- but it is also possible to confirm or to discount hypotheses". 
The Peirce definition can be compared with the very formal one used in artificial intelligence for Inductive Logic Programming (ILP) i.e. in Mugg (1999): "ILP algorithms take examples E of a concept (such as a protein family) together with background knowledge B (such as a definition of molecular dynamics) and construct a hypothesis $\mathrm{h}$ which explains $\mathrm{E}$ in terms of B". Yet, when they browse through finite sets of observations and test finite sets of hypotheses against those observations, ILP algorithms implement induction in a duly constrained mode, in which the elementary blocks of hypothesis to be induced are proposed "a priori".

In the following pages we wish to fully express the human creative capacity, and therefore use induction each time one of the two following patterns will occur:

- the inferring of a new concept (meaning an interesting collection of "features" which can be verified on a set of examples and which deserve a name "as a special aggregate") WITHOUT giving a closure to the set of all possible features;

- the inferring of a new hypothesis (meaning a well-formed proposition in a given language which can be verified in a set of situations) WITHOUT restricting the "background knowledge" with which the wellformed proposition would have to be consistent.

\subsubsection{Abduction}

The question whether abduction is just a creation of plausible hypotheses (selective abduction), or if it is inference to the best explanation, is tackled by many authors among which (Magnani 2001). In this paper, we shall consider selective abduction, even if we keep in mind that some process of verification is engaged, implying deduction and induction.

A clear pattern for abduction can be retrieved from (Peirce 1958): “Abduction is logical inference (...) having a perfectly definite logical form (...):

a. the surprising fact, $\mathrm{C}$, is observed;

b. but if $\mathrm{A}$ were true, $\mathrm{C}$ would be a matter of course,

c. hence, there is reason to suspect that $\mathrm{A}$ is true

\subsubsection{Deduction}

As for deduction, it is subject to less ambiguity for the very reason that it refers to this part of the so-called logical activity that can be entrusted to machines. Magnani (2001) gives a definition which helps to distinguish it from the two former:

"Deduction is an inference that refers to a logical implication. Deduction may be distinguished from abduction and induction on the grounds that only in deduction is the truth of the conclusion of the inference guaranteed by the truth of the premises on which it is based. Deduction refers to the so-called non-defeasible arguments."

Before ending this paragraph, let us glimpse at the view expressed in Lukasiewicz (1970), which might be a nice way to avoid long philosophical discussions about the different meanings of induction and abduction: "reasoning which starts from reasons and looks for consequences is called deduction; that which starts from consequences and looks for reasons is called reduction".

If we were to summarize the key lessons learnt in the previous chapter with the help of this new vocabulary, we might say:

- artificial agents embedded in Turing machines are purely deductive : their output is a logical consequence of their input + their program;

- only human agents have the capacity for true induction or abduction, when they start from facts and look for explanatory hypotheses.

\subsection{Rationality and Interaction}

Assuming that there is a "physical real world", involving real "objects" in which real experiences take place (therefore all chemists are looking at the same real objects and events, although they may see and therefore describe those in different ways), we shall consider "Rational Agents" independently from their human or 
artificial nature ${ }^{16}$, with the capacity to observe the "physical real world", to build theories about it, and to exchange messages about it.

In the case of EnCOrE, authors and users, senior as well as junior chemist, and Grid services, will play the role of such agents. Each agent has an "inside" and an "outside"; therefore we can sketch the following model:

- there is an interaction level where "well formed expressions" are built and recognized. At this level, "grammatical relationships among signs, independently of their interpretation or meaning" can be checked. This level is the shared syntax. Because we are concerned in this paper with Artificial Intelligence rather than by social sciences, we shall consider that any set of "well formed expressions", including a whole implemented Turing Machine, belongs to the shared syntax, as soon as the "well formed expressions" can be recognized by all the agents.

- each Rational Agent may have its private way of putting his own experiences in order, of linking some experiences to other ones, and therefore of building "meaning" upon all this processes; we shall say that each agent ${ }^{17}$ has his own private semantics. This assertion can seem a bit provocative to the reader, for it is well known that conversation, as well as cooperation, require a considerable amount of what is sometimes called "common ground". Besides, the notion of "semantics" is often used in the context of logics, when developing a "model theory" in order to give a meaning called "semantical interpretation" to logical formulas. Yet, the notion of "shared semantics" is NOT pertinent to us in the general meaning of "shared interpretation" simply because agents have no direct access to each other's way of giving a meaning to experiences: only facts belonging to the real world and descriptions of facts through a common syntax can be shared.

\subsection{Rational Agents building ontologies}

The basic "operations" in the building of ontologies with the help of shared syntax are classifying and naming: they both imply the private semantics of the agents.

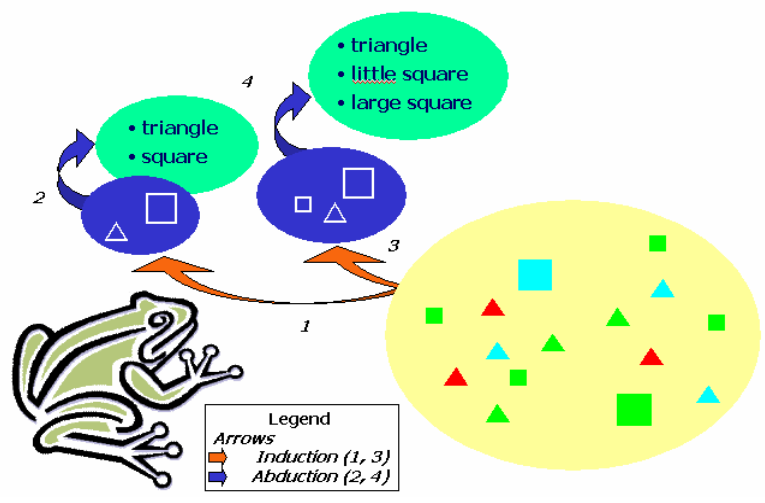

Figure 2 : Induction/Abduction cycle.

As a simplified model for any Rational Agent, this frog is able to perceive the shape of "real" objects from its "local horizon", and then to classify and name them by means of induction and abduction

Classifying happens in biological brains through the cross-activation of neural networks (Edelman 1988); it happens in the case of symbolic machine learning, for instance, through algorithmical analysis of Galois lattices (Liquière et al. 1988). The input for classification (which is merely "induction") is a set of experiences / examples, and the inductive process happens in iterative loops of induction/evaluation. In Figure 2, it is first

\footnotetext{
${ }^{16} \mathrm{We}$ are going to adopt this habit, not because we have an anthropomorphic view of software (or a mechanistic view of human thought), but rather for simplicity.

${ }^{17}$ Although we have emphasized our belief that human agents and Artificial Agents differ quite a lot concerning the ability to give a meaning to observation, we shall nevertheless accept the following viewpoint: "an Artificial Agent has its considered to have its own semantics as soon as other agents have no access to its internal program". Therefore there are two ways for a group of human agents to consider an Artificial Agent:

- either the Artificial Agent's internal processes have been validated by the group, in which case it is just a "set of validated rules" belonging to the shared syntax;

- or the Artificial Agent's internal processes are unknown from the group, and are perceived by the group as private semantics of the Artificial Agent.
} 
induced that 2 types of geometrical figures are to be considered (triangle, square), then a more accurate classification gives 3 types. This induction process takes place according to the global complexity of the agent's mind, and to the set of all his past experiments; it therefore belongs to the private semantics of the agent.

Naming makes a classification visible to other agents; otherwise it would remain confined inside each agent's private semantics. Naming is subject to evolution in accordance with the reoccurring induction. In the example given Figure 2 naming the objects "square "or "triangle" is a shortcut for asserting "objects O1, O5, O6, O7, O9 are equivalent and will be referred to as square ; objects $\mathrm{O} 2, \mathrm{O} 3, \mathrm{O} 4$ are equivalent and will be referred to as triangle". And this can be reconsidered by the agent into "objects $\mathrm{O} 1, \mathrm{O} 5, \mathrm{O} 6$ are equivalent and will be referred to as little square; objects $\mathrm{O} 7, \mathrm{O} 9$ are equivalent and will be referred to as large square; objects $\mathrm{O} 2, \mathrm{O} 3, \mathrm{O} 4$ are equivalent and will be referred to as triangle". So that naming appears as a major step in building a set of logical predicates aimed at describing the real world in the frame of a "theory". This "emission of a set of hypothesis needing validation through further experience" has been defined as "abduction".

\subsection{Rational Agents co-building theories}

Co-building theories is just another example of stabilizing a new syntactic corpus through a conversational process involving each agent's private semantics. DaNóbrega proposes several protocols ${ }^{18}$ in which the basic cycle connecting the private sphere of semantics to the public sphere of syntax is the induction/abduction cycle (DaNóbrega et al. 2002, DaNóbrega et al. 2003, and DaNóbrega 2002).

In order to be able to define unambiguous protocols for those conversational processes, let us first put the focus on a few points:

- Horizon (local experimental reality): Does each agent have a local horizon (access to only partial information) or a global horizon (access to complete information)?

- Memory: Are all the examples simultaneously given or are they sequentially introduced? How long are they stored?

- Starting point: What is the expressive power of the shared syntax?

In the following example, two agents are co-building a theory, following a protocol defined by local horizons (each one only perceives the objects included in the corresponding rectangle), simultaneous examples (we suppose that all the objects are present when the agents speak), and a shared ontology and formal language with the expressive power of first order logics (allowing IF ... THEN statements), therefore allowing deduction.

In a first step, each one induces from his own experience, and makes a hypothesis which is submitted to approval or refutation (Figure 3). In a second step, the theory has to be revised, according to the robustness of each agent's hypothesis applied inside the other's horizon (Figure 4). A subsidiary aspect of the protocol, that we leave open here, is whether the agents can "send" objects to each other in order to prove their respective assertions, or whether they simply "trust" each other (which is not a very scientific attitude!).

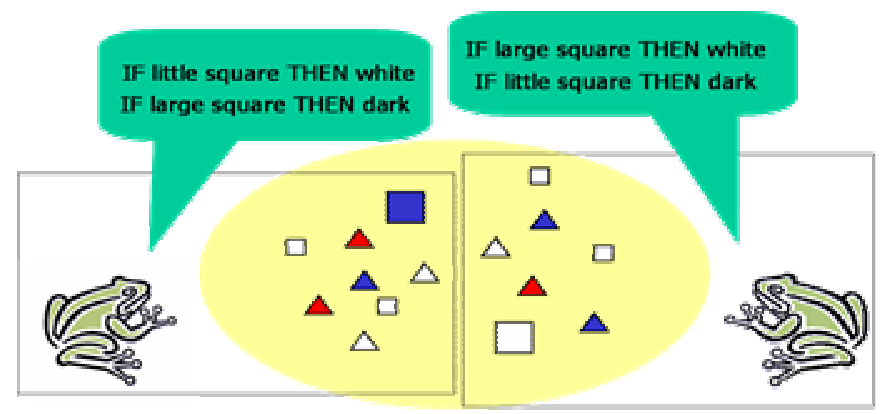

Figure 3: Agent local horizon.

Each agent makes his own abduction, according to his local horizon.

\footnotetext{
${ }^{18}$ Fidal 2000. Procédé et système de conception interactive d'une base de connaissance libre d'ambiguïtés ainsi que l'outil informatique pour la mise en oeuvre du procédé et du système. FIDAL/CNRS, Patent deposed on November, 21 2000. No. 0014999
} 


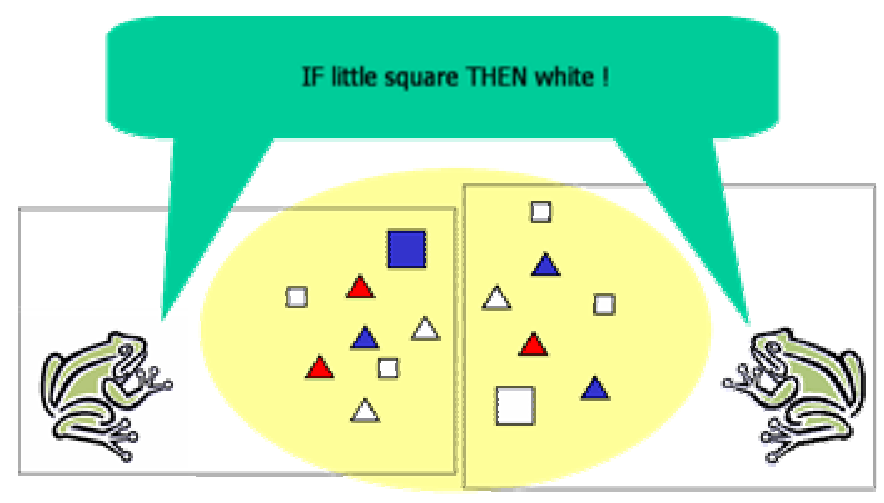

Figure 4 : Theory revision.

Each agent takes into account the other's abduction and proceeds to a revision of one's theory

\subsection{A general framework}

The diagram Figure 5 is a very simplified representation of our general framework:

- Rational Agents interpret facts into laws through induction

- then they corroborate their abductions

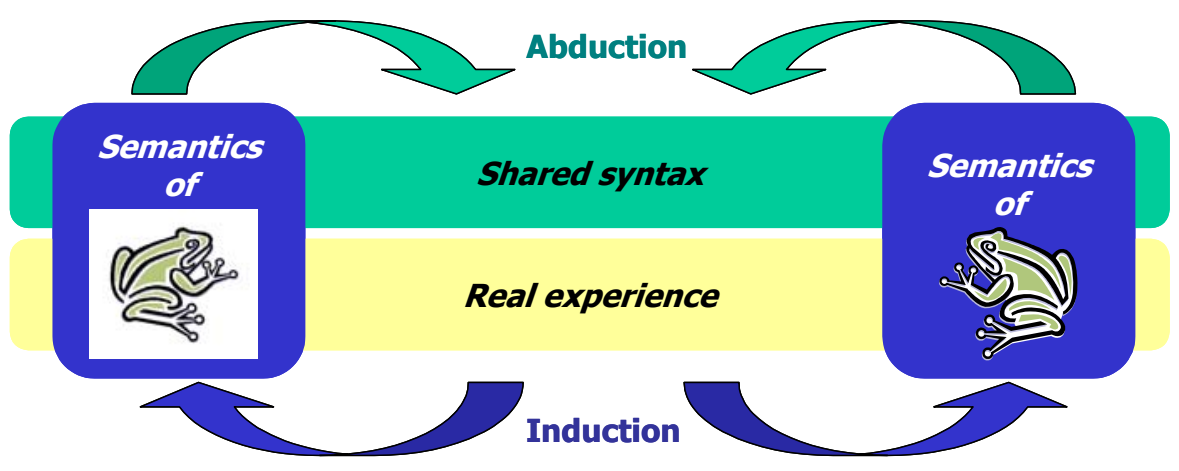

Figure 5: major flows in conceptual framework

This framework can be applied to an extension of Shapiro's approach, in which the model inference problem is solved collaboratively by the algorithm and by a group of human agents :

Let us suppose the "model inference algorithm" has been accepted by a group of agents:

this group of agents whishes to infer a model for building palindromes, starting from a collection of examples: "letusdoittiodsutel, coollooc, ..."

they have to guess the proper theoretical concepts to be included in the language, for instance "string reversal" or "string append" (this is the real difficult part !)

they run the "model inference algorithm" with different sets of theoretical concepts (input by abduction) and analyze by induction whether it succeeds or fails. Each of those experiments, although purely syntactic leads to an "objective" result that each agent can observe and from which he can induce/abduce new propositions.

\section{The interactive construction of the encyclopedia}

We are now ready to set a basis for protocols dedicated to interacting "senior" and "junior" chemists belonging to remote laboratories and willing to:

- build together a THEORETICAL CORPUS connected to an EXPERIMENTAL CORPUS via an ONTOLOGICAL NUCLEUS, powerful enough to express a deep understanding of the "chemical reality"; 
- learn from each other as a major side effect of this collaboration.

All along this collaborative process, the progressive construction of the ONTOLOGICAL NUCLEUS will be the key of theoretical progress as it will play the double role of:

- $\quad$ an increasingly powerful shared syntax;

- $\quad$ an artificial agent answering requests by pointing to experiments or to Scientific literature.

In this chapter, we first evocate the protocols implied in the interactions and the tools which will support the conversational processes, and then briefly explain and argument how the processes guided by this protocol entail learning.

Let us first question the starting point of the EnCOrE project:

- Horizon (local experimental reality): Does each agent have a local horizon (access to only partial information) or a global horizon (access to complete information)? In the context of EnCOrE, the electronic nature of the encyclopedia is a unique opportunity to merge all agents' horizons at any stage of its construction; therefore it becomes a major enabler of the collaboration. The use of a Grid Service architecture, as well as enhanced presence tools (Bachler et al. 2004), seems to us the best guarantee for a unique horizon gathering all chemists.

- Memory: Are all the observations simultaneously given or are they sequentially introduced? How long are they stored? What is in question here is the progressive elaboration of the experimental corpus. At the beginning of the process, each agent's horizon consists in his/her own bench, and a priority is to express one's own experiments in a shared syntax. We can therefore consider that the observations are sequentially introduced by the agents, in addition to an increasing experimental corpus.

- Starting point: What is the initial shared syntax? Of course, Organic Chemistry plays the role of a first level language, including objects (compounds) and laws (chemical reactions)! But we have already argued the fact that this language was not sufficient, and that ontology building was one of the major tasks to undertake in order to have a high level shared syntax. If we remember that any artificial agent validated by the group must be considered as a part of the shared syntax, two major tools at least have to be taken into consideration in the context of EnCOrE: Protégé (available at://protege.stanford.edu/) is a well known platform dedicated to ontology building, and ReSyn Assistant (Berasaluce et al. 2004; -> ReSyn) plays the role of a model inference enabler during the browsing of huge reactivity databases.

\subsection{Towards protocols for collaborative building}

Our framework for analyzing interactive theory construction is the double "abduction/induction" cycle of Figure6:

- arrow 1: from the observation of facts, scientists induce "regularities";

- arrow 2: these "regularities" are expressed in the literature, as hypothesis of "patterns of reactions" submitted to the community of chemists, which will accept or reject them;

- arrow 3: from the study of literature, scientists induce new "theoretical concepts";

- arrow 4: in order to test these theoretical concepts, they propose "experiments" that will be accepted or rejected as proofs.

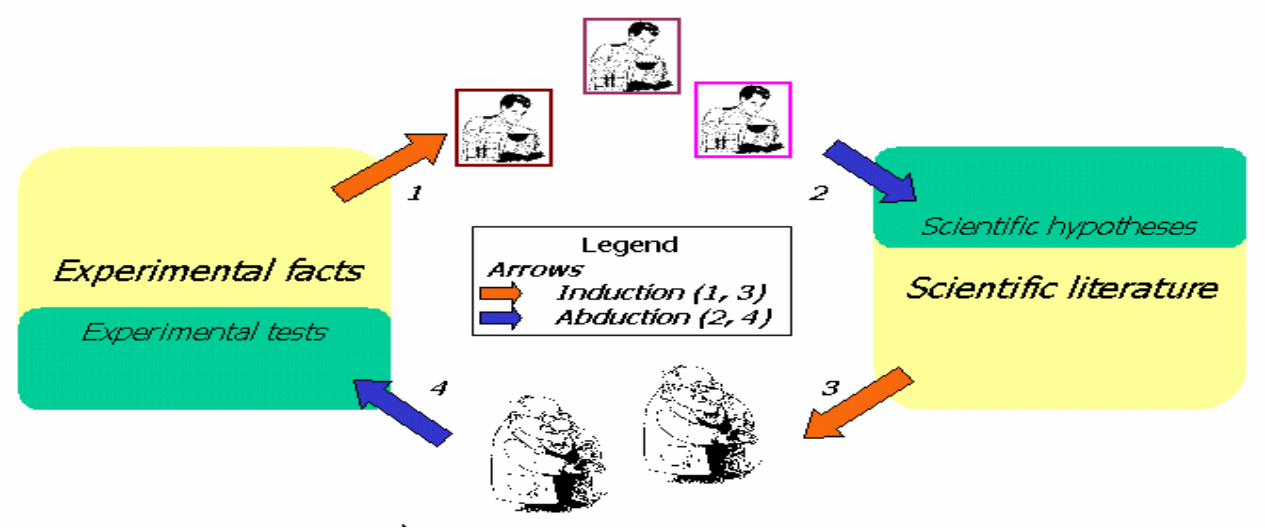




\section{Figure 6: the basic "abduction /induction" cycle}

The crucial question about the adequateness for theory formation in this kind of cycle is of course the question of convergence: whether the accumulation of experimental facts leads to an increasing consensual theoretical corpus or not. Our answer to this question, which will hopefully be experimentally verified, is that convergence relies mainly on three factors:

1. intersection of horizons realized through capitalization into an electronic medium as well as communication with the help of "enhanced presence" tools;

2. increasing collective memory of facts, which is mainly correlated to the building of the experimental corpus;

3. well defined PROTOCOLS in order to rule the conversational processes which will occur during "theory revision"; such protocols rely on a little number of roles to be played by the agents (Author, Editor, Partner, Reviewer ...) and take into account the FIPA-ACL ${ }^{19}$ performatives.

When building the experimental and the theoretical corpus, the focus will be put on collaborative work between scientists through discussions, controversies and consensus phases. We intent to perform experimental learning through access to dialogues related to the construction of the encyclopaedia. We also imagine allowing the "learner" to have access, via EnCOrE, to sessions in which a teacher, a learner and a scientist interact on a specific subject. Figure 7 illustrates the main conversational processes:

- EXPERIMENTAL CORPUS: Juniors build inductive reasoning (arrow 1a) about experimental facts, ABDUCE new experiments aimed at infirming or confirming their interpretation, and as soon as this local cycle is stabilized, abduct descriptions of these experiments at the syntactic level (arrow 2a) in the reactivity database, using terms from the ONTOLOGICAL NUCLEUS.

- THEORETICAL CORPUS: On the basis of their respective reading of the scientific literature ${ }^{20}$, Seniors build inductive reasoning (arrow 1b) about what has been written in the past by themselves or others, and submit (abduction arrow 2b) theoretical hypothesis inside articles structured in reference to the pragmatic points of view listed in the ONTOLOGICAL NUCLEUS.

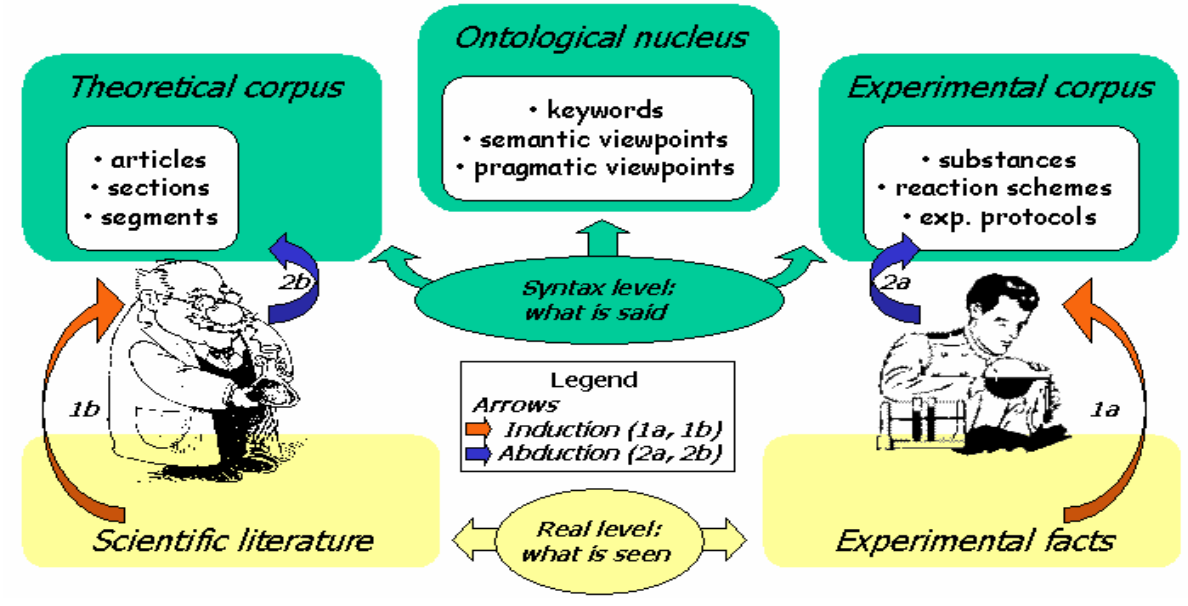

Figure 7: Experimental facts and scientific literature

The protocols we have mentioned will rely on strong interaction between a great numbers of contributors. If "juniors" may appear willingly involved in such constructive interactions, seniors are expected to have little time to spend...that is why this kind of protocols would remain purely theoretical without the help of COMMUNICATION TOOLS (e mail, discussion forums, instant messaging, audio/video conferences), as well as

\footnotetext{
${ }^{19}$ Agent Communication Languages adopted by the Foudation for Intelligent Physical Agents

${ }^{20}$ Although it is of syntactic nature, the scientific literature automatically acquires the status of "shared reality": we do not mean that different chemists interpret what they read in the same way (if so, there would be no problem at all), we just mean that they read the same sentences.
} 
INFORMATION MANAGEMENT TOOLS (collaborative edition of documents/schemes, ...) allowing a robust capitalization upon asynchronous conversations and distant interaction based on enhanced presence.

The choice of a GRID SERVICE ARCHITECTURE incorporating all those TOOLS is not based upon the need for power (which is not critical here except when running heavy Molecular Dynamics or Molecular Mechanics calculations) It is based on the simple observation that the basic needs of any Virtual Community are :

a feeling of "home" (easy connection, mutual trust, security)

the easy capacity of self-management of members \& tools/services

And these needs match exactly what Grids will offer:
a global Security Infrastructure
technology-transparent management of services (including members management)
a easy access to any service instance through a unique identifier (for an individual use of a tool as well as for a group videoconference)

All the collaborative constructions imply the progressive set-up of a system with memory of interaction (between Author and Editor, Author and Partner, Reader and Helper, etc.) which will allow us to study the conversational process in order to improve remote interactions about ontology building (agent roles, typical use cases). In a future step, it is imagined to set-up an agent to assist in ontology building by using previous interactions. The system currently under design has the following requirements:
effective collaboration between agents
enhanced knowledge sharing
instant help providing
enhanced controversy expression

\subsection{Towards protocols for Learning}

EnCOrE intents to favour learning in Organic Chemistry intuitively, at any time, at any age, at any level from beginner to highly educated research scientists. The priority is that interactions exist and are facilitated by technologies with respect to a shared construction of meaning. Therefore, although learner models are necessary tools, the current focus is on enhanced remote and constructive interactions.

According to Bourdeau et al. (2002), shared intelligence among the designer/author and the authoring environment is the dynamic component for the interactive construction of instructional scenarios and learning environments. Therefore, declarative knowledge about instruction could be the "beating heart" of an Intelligent Tutoring System. In the context of the EleGI / EnCOrE project, the operational principle of building an instructional system that sustains and fosters the learning process will be in our focus during the analysis of conversational processes, and the designing of tools for enhanced presence.

Our intention will not be to investigate speech acts produced by "teachers" against the effects they may have on students learning, as it is done in Porayska-Pomsta et al. (2000), in reference to a traditional "teaching paradigm". It will be to investigate dialogue, through dialectic processes exemplified by the tutor and internalized by the learner, because such processes guide the learner's reasoning and accelerate the transition from naïve to scientific conceptualizations. Therefore our work will be inspired, among other ones, by Ravenscroft et al. (2000).

Collaborative learning will happen during the whole building cycle of the encyclopedia (Figure 8):

- When describing experimental facts in the terms of the ontological nucleus (arrows $1 \mathrm{~b}, 2 \mathrm{~b}$ ) and indexing them, a peer-to-peer based virtual laboratory environment should allow sharing of communication and collaboration activities, in particular to solve the difficulties in discussion about hardly printable information resources like an ontology in Protégé, or an analysis made by ReSyn Assistant (available at://www.lirmm.fr/ resyn/presresyn.html).

- When structuring scientific articles according to the ontological nucleus, splitting them according to pragmatic viewpoints (arrows 1a, 2a) and indexing them, forums will be attached to each article and to the dictionary. Readers, Authors, Editors will contribute to the discussion about the dictionary use, structure, and content, about the sense defined in the articles. These interaction traces will allow authors and editors to correct and to adapt the content to the reader, to adjust the service to meet with user behavior. 
- The editors will moderate public fora, observe "indexation processes" interfere and update the ontological nucleus (arrows 1c, 2c, 1d)

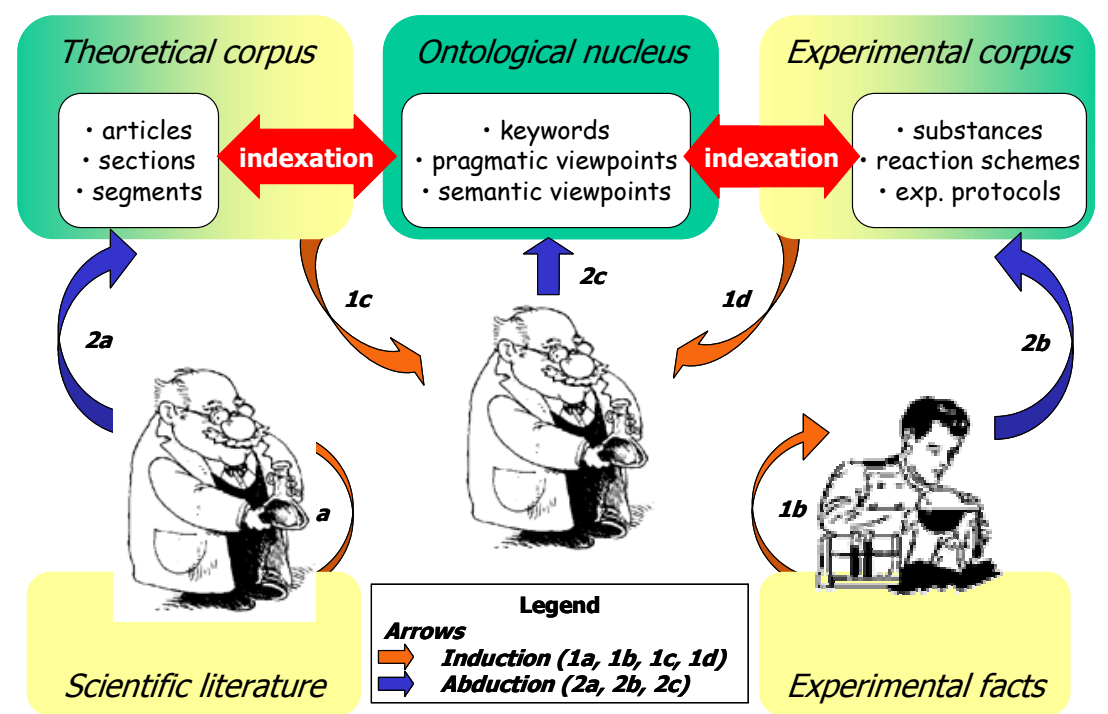

Figure 8: Learning while building the encyclopedia

Active learning will happen when using the encyclopedia and questioning the theoretical corpus (Figure 9):

- Seniors provide articles (arrow $2 \mathrm{~b}$ ) in the theoretical corpus, which are structured according to pragmatic viewpoints (arrow 1b) in order to assist searching and reading of the encyclopedia;

- Juniors ask questions about the theoretical corpus (arrow 1a) and therefore cause specific pragmatic viewpoints to appear (arrow 2a).

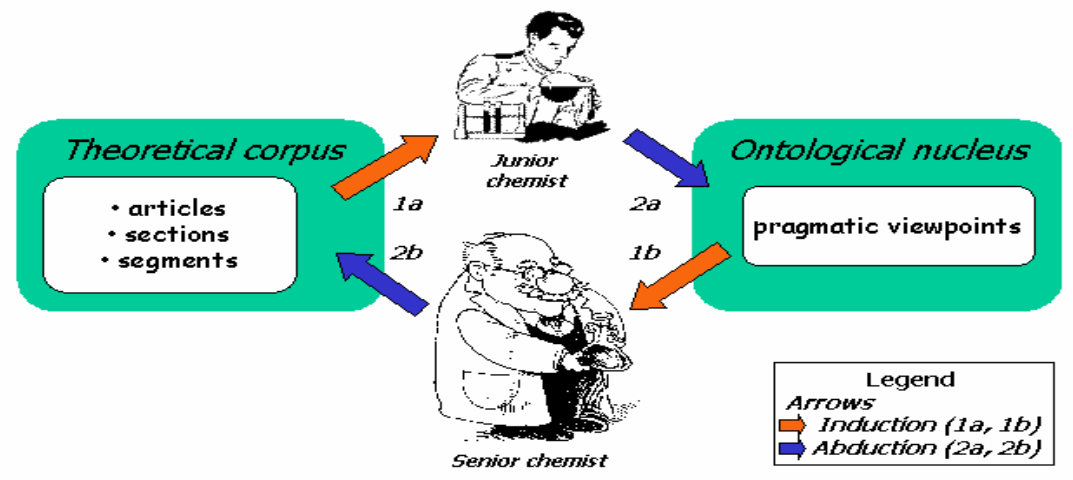

Figure 9: Learning while using the encyclopedia

\section{Conclusions and perspectives}

Theory building, as well as Human learning are hard subjects, to understand and to foster. Enhancing Technologies have often adopted a classical paradigm emerging from the recent history of teaching, more than on profound considerations - such as those reported in the "Dialogues" of Plato's conversations with his teacher Socrates - that would have required to enter into the subtleties of human behavior, before producing any technical aid.

We have described above the first phase of an ambitious research work that aims at a deeper exploration of human behavior when humans are engaged in a common challenge: build collaboratively an Encyclopedia for Organic Chemistry. Human learning, in that endeavor, is mixed with human understanding and human scientific work. 
The paper has shown that it is possible, with the currently available technologies, to foresee a generic yet quite neatly defined architecture supporting understanding, doing and learning within remotely connected virtual Communities interacting in the Grid, which we understand as a network of human and artificial agents exchanging services. The lessons we have learned from this study, and that we present and exemplify in the paper are mainly two:

1. understanding, doing and learning occurs as chains of deductions, inductions and abductions that reflect quite directly our common experience in reasoning, abstracting and generalizing on and from examples, on and from conceptualizations. The difference with many other attempts is that these operations are put into the social perspective of conversations for a common goal. This rational aspect of the processes within constructive interactions may be today represented, controlled and enhanced by suitably designed computational structures organized within an architecture equipped with conversational protocols. Previous experiences performed also by some of us in different depth and domains have shown the feasibility and the adequateness of the approach. What will be challenging for us now is the leap in scale and the genericity of the model, and its corresponding realizations.

2. understanding, doing and learning are not purely rational activities, but require emotional aspects, such as motivation, trust, confidence etc. Our objective here is not to monitor such aspects, but only to help and integrate them. The conversational approach we have described above, together with the enhanced presence tools we just have cited in passing, and the analysis of conversational traces, argumentations, contradictions and dynamic human help available online seem to us very promising for becoming a crucial support offered today by technologies.

We did not enter these last issues in depth in the paper, as the first one - the architecture - was already dense and required to perform and justify quite selective choices. However, we are perfectly aware that if there will be one bottleneck in the EnCOrE project, this will be due to emotional aspects not sufficiently cared for by the initial versions of the system. Therefore, we will address these issues as the most important ones deserving our priority in future activities in the EnCOrE project. 


\section{Annex: a long quote from Mario Alai (ALAI 2004)}

"In (Langley et al. 1987), BACON 4 (one of those programs intended to mechanize scientific discovery) can deal with cases in which data are names of objects ...when the values of a given property of one object (e.g. the current of one battery) vary with the related objects (e.g. with different wires), then it must be postulated that there is a property of these related objects which is responsible for such variation (e.g., conductance), and whose values are proportional to those of the first property (current) ....The authors stress that this amounts to the introduction of theoretical, i.e. unobservable, properties ....Nonetheless, this does not seem to be a full-fledged example of theorization, since the new property is not embedded in a whole theory describing it, its nature, causes, functioning, effects, etc. Its introduction, here, is just matter of detecting a regularity in the behavior of observable objects and properties, and ascribing to it as a cause a property which is identified just by means of this particular effect ... Another heuristic tells BACON$_{4}$ to look for common divisors and their regularities. In this way the program may be applied to chemical research, and starting from the proportions of weights and volumes of elements in compounds it finds the molecular and atomic weights of various elements and compounds. This is not to say it discovers either the molecular or atomic theory, however; for it finds just numbers, and it is only in the light of our knowledge of atomistic chemistry that we may interpret those numbers as atomic or molecular weights. The last version, BACON ${ }_{5}$, was created by adding to the earlier ones the notion of physical symmetry, and the rule that if a particular relation holds among a set of variables, (e.g., two objects with respective initial and final velocities), it must be presumed that it holds among variables of the same type (e.g., two different objects with respective initial and final velocities). Hence, on condition of being told which variables are of the same type, the system is able to speed up significantly its search for regularities (such as the law of conservation of momentum, Snell's law of refraction, or Joule's law). BACON ${ }_{5}$ is thus theory-driven, i.e. it imitates those cases of actual science in which research is not merely based on data, but on theoretic presuppositions as well. Even more theory-driven is a different system, BLACK, which has inbuilt the notion that certain properties are conserved... starting from data about reactions, and assuming that the number of molecules involved in a reaction is proportional to the volumes of the respective elements, DALTON infers the molecular structure of compounds. Moreover, assuming that atoms are conserved in reactions, and that molecules are composed of the smallest number of atoms compatible with the law of conservation and the known molecular structures, DALTON infers the atomic structures of elements and compounds.

... Donald Gillies contrasts them (the results of Simon's work) with the programs produced by disciples of Alan Turing and researchers working in their tradition, such as Ehud Shapiro, Stephen Muggleton, Donald Michie, Edward Feigenbaum, Bruce Buchanan, J.R. Quinland and Ivan Bratko, programs which have successfully been applied to the solution of practical problems and have made new (hence actual) discoveries ... For instance, DENDRAL, developed since 1965, accomplishes what an expert chemist might do, inferring the molecular structure of organic compounds from their mass spectrogram. ASSISTANT has been able to diagnose various kinds of disease better than human specialists. GOLEM was able in 1991 to predict the secondary structure of proteins from their primary structure... Gillies credits such rules as "new laws of nature" discovered by golem. But it is far from clear that they qualify as such, because (a) they have a very low level of generality, as the above example shows, (b) as he himself concedes they fail in about $20 \%$ of cases, and therefore (c) it is dubious that they describe actual causal relationships, as opposed to contingent statistical correlations... all these programs (DENDRAL, ASSISTANT, GOLEM) produce generalizations connecting a target property (such as a secondary structure character, or a particular disease), to the presence or absence of symptomatic properties (such as primary structure characters, or symptoms manifested by patients); they do so by checking which symptomatic properties are present or absent when the target property is known to be present. This is to say, they practice, though with sophisticatedly iterated procedures, nothing but Baconian or Millian induction. No wonder therefore these programs have only discovered low level generalizations, and no theories, theoretical laws, entities or explanations. Just as enumerative induction, also Bacon's tables and Mill's canons, in fact, may establish horizontal links among empirically known entities or properties, but no vertical links among observable and non-observable levels of reality."

An example (of different approach) could be the proposal advanced by John Holland, Keith Holyoak, Richard Nisbett and Paul Thagard (henceforth HHNT) in (Holland et al. 1986). They try to reconstruct the cognitive processes by jointly relying on cognitive science, philosophy and computer science, and describe a program ("Pl", for "processes of induction") which purports to replicate the crucial features of such processes.

Knowledge is represented by HHNT as the construction of mental models of the environment. Environment, in turn, is described as consisting of states and transition functions among them ... there can be long chains of states and transition functions, and particularly important chains are those in which the initial state represents a theoretical or practical problem for the knowing subject, and the final state represents its solution (HHNT stress the importance of pragmatics in their approach). For HHNT the mind is like a bulletin board, on which "messages" (i.e. propositions) are posted ...In mental models states are represented by categoric messages (e.g.: "all bodies move"; "this body is moving"; etc.), and transition functions by hypothetical messages, or "rules" (e.g., "if a body moves at time $t$, it will stand still at time t'")...As it happens, however, there is only limited room on a bulletin board, as well as in the human mind. Therefore, not all potentially activated messages will be posted, and messages will compete for room on the board. Besides, mutually incompatible messages that might be posted will be in competition even independently of room scarcity. The competition takes place more or less as it happens on the economic market: messages and rules in a chain can be viewed as suppliers, middlemen and consumers, where selling and buying consists in activating or being activated. When the final ring of a chain constitutes the successful solution to a problem (hence the importance of the pragmatic dimension in this approach), its success is comparable to profit, which is duly shared with each preceding ring, as each buyer pays back the goods or services that reached him through the chain ... In practice, the environment's feed-back gradually reinforces successful beliefs and extinguishes unsuccessful ones, just like market reactions make efficient firms flourish and inefficient ones go bankrupt... Admittedly, PI, HHNT's program, has not (yet) made any new discovery, nor any old one as complex and important as those achieved by Simon's programs." 


\section{References}

Alai M. 2004. A.I., Scientific Discovery and Realism, Minds and Machines, Volume 14, Issue 1. February pp: 21 - 42 Bachelard G. 1975. La formation de l'esprit scientifique, Vrin, Paris

Berasaluce, S., Laurenço, C. Napoli A., Niel G. 2004. An experiment on knowledge discovery in chemical databases. Lecture Notes in Artificial Intelligence, vol. 3202, 39-51

Bourdeau, J., Mizoguchi, R. June 5-8 2002. Collaborative Ontological Engineering of Instructional Design Knowledge for an ITS Authoring Environment. In S.A. Cerri, G. Gouardères, and F. Paraguaçu, editors, Intelligent Tutoring Systems 6th International Conference - ITS 2002, Biarritz (France), LNCS 2363, pp. 399-409. Springer-Verlag Berlin Heidelberg.

Bachler, M, Buckingham Shum, S., Chen-Burger et al. 2004. Collaboration in the Semantic Grid: a Basis for e-Learning'. In:7th Int. Conf. On Intelligent Tutoring Systems (ITS 2004)-Workshop on GRID Learning Services (GLS04). Best paper award. Summary available at http://www.info.uqam.ca/ nkambou/gls/gls04.htm Extended version to be submitted to: Applied Artificial Intelligence Journal, special issue on Learning Grid Services, forthcoming

Bravo, C.,Redondo, M. A., Ortega, M. et al. June 5-8 2002. Collaborative Discovery Learning of Model Design. In S.A. Cerri, G. Gouardères, and F. Paraguaçu, editors, Intelligent Tutoring Systems 6th International Conference - ITS 2002, Biarritz (France), LNCS 2363, pp. 671-680. Springer-Verlag Berlin Heidelberg.

Chen L, Shadbolt N.R., Goble C. et al. 2003. Towards a Knowledge-based Approach to Semantic Service Composition. 2nd International Semantic Web Conference (ISWC2003) 20-23 October, Florida, USA. Lecture Notes in Computer Science, LNCS 2870, pp 319-334.

Cerri, S. A., Cheli, E. \& McIntyre, A. 1990. NOBILE: User Model Acquisition in a Natural Laboratory. In Proceedings of NATO ASI on Synthesis of Instructional Science and Computing Science, Calgary

Cerri, S. A., Eisenstadt, M., Jonquet, C. 2005. Dynamic Learning Agents and Enhanced Presence on the Grid. Towards the Learning GRID: advances in Human Learning Services" IOS Press, Amsterdam.

Cerri, S. A. Open Learning Service Scenarios on GRIDs. 2005. Towards the Learning GRID: advances in Human Learning Services" IOS Press, Amsterdam.

Colaux-Castillo, C., Krief, A. 2005. EnCOrE (Encyclopédie de Chimie Organique Electronique): an Original Way to Represent and Transfer Knowledge from Freshmen to Researchers in Organic Chemistry.Towards the Learning GRID: advances in Human Learning Services" IOS Press, Amsterdam.

Crozat, S., Trigano, P. June 19-23 2000. An ontological approach for design and evaluation of tutoring systems. Gilles Gauthier, Claude Frasson, Kurt VanLehn (Eds.): Intelligent Tutoring Systems, 5th International Conference, ITS 2000, Montréal, Canada, Lecture Notes in Computer Science 1839. pp. 651. Springer-Verlag Berlin Heidelberg.

Crubézy, M. and Musen, M.A. 2003. Ontologies in Support of Problem Solving. Handbook on Ontologies.S. Staab and R. Studer, Springer-Verlag Berlin Heidelberg. pp: 321-341

DaNóbrega. 2002. Une Approche Dialectique à la Formation de Théories : Aspects Conceptuels; Formels et Pragmatiques dans le Cadre de l'Apprendissage Humain. Thése de Doctorat. Université Montpellier II..Montpellier.

DaNóbrega, G. M., Cerri, S. A., Sallantin, J. June 5-8 2002. On the Social Rational Mirror: learning e-commerce in a Webserved Learning Environment. In S.A. Cerri, G. Gouardères, and F. Paraguaçu, editors, Intelligent Tutoring Systems 6th International Conference - ITS 2002, Biarritz (France), LNCS 2363, pp. 41-50. Springer-Verlag Berlin Heidelberg

DaNóbrega, G. M., Cerri, S. A., Sallantin, J. 2003. A Contradiction-driven Approach of Learning in Discovery Learning Environments SBIE'03:XIV Simposio de Informatica na Educaçcao, pp. 453-462 Best paper award (out of 330). Journal of the Brazilian Computer Society.

Duquenne. 1999. Latticial structures in data analysis. Elsevier Science B.V.

Fensel, D., Harmelen, F. van, Ding,Y; et al. 2003. On-To-Knowledge: Semantic Web Enabled Knowledge Management. In IEEE Computer

Ferber, J. 1999. "Multi Agents Systems: an introduction to distributed artificial intelligence". Addison-Wesley

Edelman, G. M. 1988. "Neural Darwinism: The Theory of Neuronal Group Selection”. Basic Books, New York.

Gold, E. M. 1967. Language identification in the limit. Inform. Control, 10, 447-474,

Gómez-Pérez, A. 1999. Ontological Engineering: A State Of The Art . Expert UPDATE, 2(3): 33-43

Gruber, T. R. 1993. A. Translation Approach to Portable Ontology Specifications. Knowledge Acquisition, 5(2), , pp. 199-220

Guzdial, M., Kolodner, J., Hmelo et al, Newstetter, W. 1996. Computer support for learning through complex problem solving Communications of the ACM. Volume 39, Issue 4 : pp: 43 - 45.

Holland, J., Holyoak, K., Nisbett et al. P. 1986. "Induction. Processes of Inference, Learning and Discovery". MIT Press, Cambridge, Mass., London, England

IUPAC \{International Union of Pure and Applied Chemistry\} Compendium of Chemical Terminology, Second edition 1997, Edited by A D McNaught and A Wilkinson. http://www.iupac.org/publications/compendium/index.html

Klein, M., Fensel, D. 2001. Ontology versioning for the Semantic Web. In Proceedings of the First International Semantic Web Working Symposium (SWWS).Stanford University, California, USA.. pp: 75-91.

Kuhn, T.S. 1970. The Structure of Scientific Revolutions. University of Chicago Press

Knublauch, H. 2003. An AI tool for the real world: Knowledge modeling with Protégé. Java World Journal, June. (To be downloaded from : http://www.javaworld.com/javaworld/jw-06-2003/jw-0620-protege.html on 20/01/2004). 
Langley,P., Simon, H.,Bradshaw, G., Zytkow,J. 1987. Scientific Discovery.Computational Explorations of Creative Processes. MIT Press, Cambridge, Mass., London, England.

Langley,P. 2000. The computational Support for Scientific Discovery. International Journal of Human-Computer Studies, 53, 393-410.

Lavoisier, A-L. 1789. Traité Élémentaire de Chimie. Cuchet, Paris.

Lemoisson, P., Cerri, S. A., Sallantin, J. 2005. Conversational Interactions of Rational agents. Towards the Learning GRID: advances in Human Learning Services" IOS Press, Amsterdam.

Liquière, M., Sallantin, J. 1998. Structural machine learning with gallois lattice and graphs. In $5^{\text {th }}$ International Conference on Machine Learning, pages 305-313, Madison, Wisconsin (USA), Morgan Kaufmann.

Lukasiewicz, J. 1970. Creative elements in science 1912 in J. Lukasiewicz, Selected Works, North Holland, Amsterdam, pp $12-44$

Magnani, L. 2001. Abduction, Reason and Science / Processes of Discovery and Explanantion. Kluwer Academic/Plenum Publishers

Mill, J. S. 1843. Système de logique deductive et inductive. 1843. Livre III " de l'induction » (available at http://www.uqac.uquebec.ca/zone30/Classiques_des_sciences_sociales/classiques/Mill_john_stuart/systeme_logique/livre_3/ systeme_logique_3.html)

Muggleton, S. 1999. Inductive Logic Programming: Issues, results and the challenge of Learning Language in Logic. Artificial Intelligence 114 (1999) 283-296

Noy, N.F., Sintek, M., Decker, S., Crubézy, M. et al. 2000. Creating Semantic Web Contents with Protégé-2000. In IEEE Intelligent Systems, Vol. 16, No. 2, March/April 2001, special issue on Semantic Web, pp. 60-71.

Noy, N. F. and Klein, M. July 2004. Ontology evolution: Not the same as schema evolution. In Knowledge and Information Systems, Volume 6, Number 4, pp. 428 - 440. Springer-Verlag London.

Piaget J. 1968. Sagesse et illusions de la philosophie PUF, Paris

Peirce, C. S. 1955 "Philosophical writings of Peirce" .J; Buchler ed, Dover, New York,

Pierce, C. S. 1958. Collected Papers, 8 vols, C. Harshorne and P. Weiss (vols. I-VI), and A.W. Burks (vols VII-VIII), ed Harvard University Press, Cambridge MA 1931-1958

Popper, K. R. 1959. The Logic of Scientific Discovery. Hutchinson, London, New York

Porayska-Pomsta, K., Mellish, C., Pain, H. 2000. Aspects of Speech Act Categorisation: Towards Generating Teachers Language. International Journal of Artificial Intelligence in Education. Volume 11, 254-272.

Ravenscroft, A., Pilkington, R. M. 2000. Investigating by design: developing dialogue models to support reasoning and conceptual change. International Journal of Artificial Intelligence in Education: Part I of the Special Issue on Analysing Educational Dialogue Analysis. Edition, 11, 3, 273-299

Shapiro, E. 1991. Inductive inference of theories from facts/ Computational logic (essays in honour of Alan Robinson) Chap7, edited by Jean-Louis Lassez and Gordon Plotkin, The MIT Press

Stojanovic, L., Maedche, A., Motik et al. 2002 User-driven Ontology Evolution Management. Proceedings of the 13th European Conference on Knowledge Engineering and Knowledge Management EKAW, Madrid, Spain

VonGlasersfeld, E. 1978. Radical constructivism and Piaget's concept of knowledge. In F. B. Murray (Ed.), The impact of Piagetian theory (pp. 109-122). Baltimore, MD: University Park Press.

Vygotsky L.S. 1978. Mind in Society. The Development of Higher Psychological Processes. Edited by M. Cole, V. JohnSteiner, S. Scribner and E. Souberman.Harvard University Press. Cambridge, Massachussets

Yoshinobu, K., Masakazu , K., Masayoshi, F. et al. 2004. Deployment of an Ontological Framework of Functional Design Knowledge. Advanced Engineering Informatics 